\title{
ASPECTS OF THE THEORY OF MIND IN THE INSIGHT INTO THE DISEASE IN PATIENTS WITH SCHIZOPHRENIA
}

\author{
Simona Trifu, University of Medicine and Pharmacy "Carol Davila" Bucharest \\ Ana Miruna Dragoi, Clinical Hospital for Psychiatry "Alex. Obregia" \\ Antonia Ioana Trifu, Medical Military Institute, General Medicine, Bucharest, Romania
}

In the case of the first hypothesis (schizophrenic patients will have significantly lower scores than the control group), the independent variable is constituted by the presence or absence of the psychic disorder. Thus, the independent variable is a nominal dichotomous one.

The dependent variable refers to the scores obtained in the Theory of Mind tests. By schematizing, the variables of the first hypothesis are:

V.I.1 = People with schizophrenia (1) Non-schizophrenic persons $(0)$;

V.D.1 $=$ The scores obtained in the Theory of Mind tests.

The independent variable for the following hypotheses is the score obtained by patients suffering from schizophrenia in the Theory of Mind tests. The dependent variables are: Clinical Insight score, Cognitive Insight score, Self-Certainty score, SelfReflection score. In view of these facts, the dependent variables are:

V.I.2= Scores obtained in the Theory of Mind tests;

V.D.2= Clinical Insight Score;

V.D.3 = Cognitive Insight Score;

V.D. $4=$ Self-Certainty score;

V.D. $5=$ Self-Reflection score.

For the following five hypotheses, the independent variable is the Cognitive Insight, and the dependent variables are: Depression, Conceptual Disorganization, Affective Flattening, Stereotypical Thinking, and Delirium. Thus, the following variables are:

V.D.6 = Score obtained on Depression;

V.D.7 = Score obtained on Conceptual Disorganization;

V.D.8= Score obtained on Affective Flattening;

V.D.9= Score obtained on Stereotypical Thinking;

V.D. $10=$ Score obtained on Delirium.

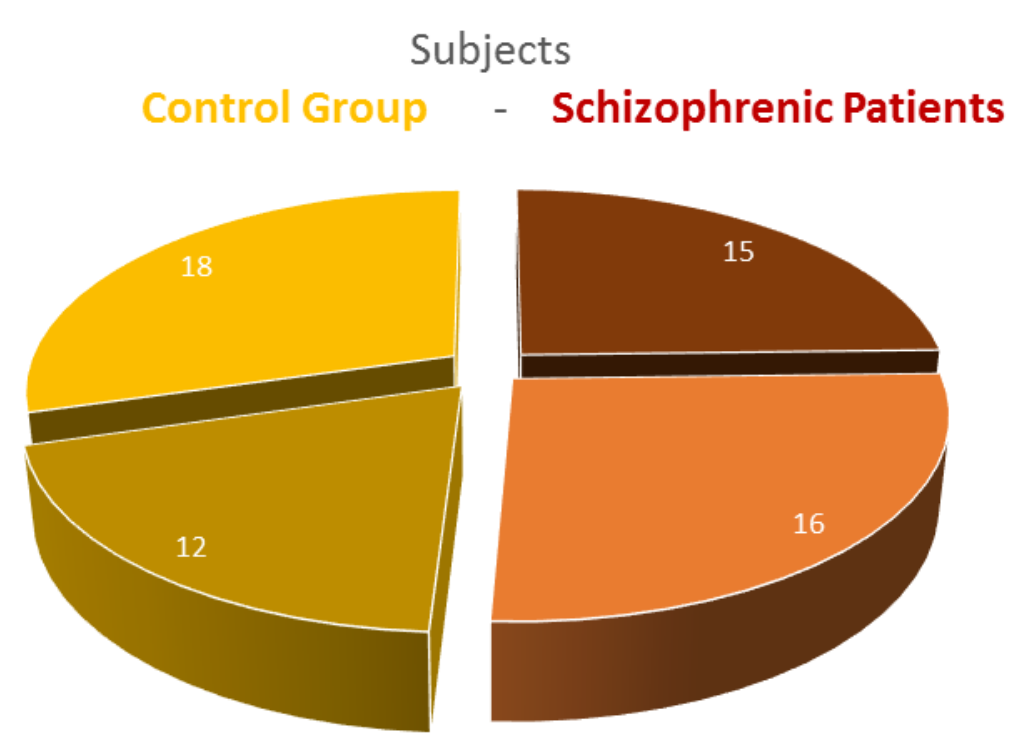

- Male - Female - Male a Female

Tools:

The Beck Cognitive Insight Scale has been used to measure the Cognitive Insight focusing on cognitive processes involved in the re-assessment of abnormal situations experienced by schizophrenic patients and their specific interpretation, aiming at: distancing, objectivism, perspective and self-correction

To score the Theory of Mind, it was used a tool developed by Frith [6] consisting of six short stories that require the inference of the mental states of the characters and which gradually become more complex $(\alpha=.85)$.

The measurement of positive / negative symptomatology was performed using the Positive and Negative Syndrome Scale (PANSS)

\section{RESULTS:}

The ToM abilities of schizophrenic patients $(m=5.48 ; s=5.27)$ differ significantly from the level of ToM abilities of the nonclinical group $(m=16.00 ; s=2.27), t=-10.16, \quad p<.001$. The confidence interval limits (95\%) range from -12.60 to -8.42 .

- The more the schizophrenic patients will have lower scores on the ToM (deficit), the more they will have a higher (severe) score on the Clinical Insight scale. The statistical data obtained suggest that there is a substantial negative correlation between the mentalization abilities and the Clinical Insight scale, thus $r(29)=-.62, p<.000$.

- The higher the schizophrenic patients will have lower scores on the ToM (deficit), the lower the score on the Cognitive Insight scale will be. The statistical data obtained suggest that there is a significant positive correlation between mentalization abilities, and the Cognitive Insight scale measured by the BCIS scale, thus $r(29)=.67, p<.001$.

- The more the schizophrenic patients will have the lowest scores on the ToM (deficit), the lower the score for SelfReflection on the Cognitive Insight scale. The statistical data obtained claim that there is a significant positive correlation between the mentalization abilities, and the Self-Reflection measured by the BCIS scale, thus $r(29)=.63, p<.001$

- The more the schizophrenic patients will have lower scores on the ToM (deficit), the higher the score on the Self-Certainty in the Cognitive Insight scale. The statistical data obtained suggest that there is a moderate negative correlation between the mentalization abilities, and the Self-Certainty measured by the $\mathrm{BCIS}$ scale, thus $\mathrm{r}(29)=-.49, \mathrm{p}=.005$.

- the existence of a correlation between the Cognitive Insight and the Conceptual Disorganization described in PANSS. The statistical data obtained support that there is a substantial significant negative correlation between the two variables, reporting $r(29)=-.51, p=.003$.

- The correlation between the Cognitive Insight and the Affective Flattening scale described in PANSS - the statistical data does not support the existence of a correlation between the two variables, reporting $\mathrm{p}=.13$

the existence of a negative correlation between the Cognitive Insight and the Stereotypical Thinking scale described in PANSS. The statistical data obtained support that there is a substantial negative correlation between the two, thus $r(29)=-.60, p<.001$

\section{DISCUTIONS:}

\section{Limitations}

- there is a plethora of tests that measure the ToM, which vary in verbal abilities and the affective processing needed to fill out. That being said, we cannot say exactly to what extent the chosen tests cover the range of ToM dimensions.

- the exclusion criteria narrowed the response range of several items in the PANSS scale. Thus, in the case of Conceptual Disorganization, scores 6 and 7 impose deterioration until the abolition of communication skills in an interview (6: thinking is seriously derailed and inconsistent / incoherent internally, resulting in major irrelevancies and disturbances of thinking processes, that occur almost constantly; 7: thoughts are disturbed until the patient's incoherence. There is a marked weakening of associations, resulting in the total inability to communicate).

- the status of the disorder was not controlled (acute / chronic / remission), the duration of the disease, the severity of the symptoms.

- the Clinical Insight was assessed through a single item of the PANSS test. Although this item has been used as a method of assessment by many researchers [19], it is advisable to find a complex assessment method. 